\title{
AN ANALYSIS OF COORDINATED AND NON-COORDINATED MEDIUM ACCESS CONTROL PROTOCOLS UNDER CHANNEL NOISE
}

\author{
Tolga Numanoglu, Bulent Tavli, and Wendi Heinzelman \\ Department of Electrical and Computer Engineering \\ University of Rochester, Rochester, NY 14627 USA \\ Email:\{numanogl, tavli, wheinzel\}@ece.rochester.edu
}

\begin{abstract}
In this paper, we present an analysis of the effects of channel noise on the performance of coordinated and noncoordinated MAC protocols. In order to observe the degradation in the performance of a coordinated MAC protocol (MH-TRACE) with increasing BER level, we created an analytical model to estimate MH-TRACE's performance. This analytical model is validated through simulation experiments. Our results show that despite its higher level of vulnerability, the coordinated MAC protocol's performance loss is comparable to the performance loss of the noncoordinated MAC protocol (IEEE 802.11) for low to mid $B E R$ levels (i.e., $\left.B E R<10^{-4}\right)$. On the other hand, for extremely high $B E R$ levels (i.e., $B E R \geq 10^{-4}$ ) the performance loss of the coordinated MAC protocol is comparatively higher than the performance loss of the non-coordinated MAC protocol due to its dependence on control traffic, which is also affected by the BER level.
\end{abstract}

\section{INTRODUCTION}

Medium Access Control (MAC) protocols are employed to control access to the channel in order to regulate transmissions to avoid or minimize collisions [1]. Furthermore, the MAC protocol is the key element in determining many features of a wireless network, such as throughput, Quality of Service (QoS), energy dissipation, fairness, stability, and robustness [2], [3], [4]. In other words, the performance of a particular network highly depends on the choice of the MAC protocol.

MAC protocols can be classified into two categories based on the collaboration level of the network in regulating the channel access: coordinated and non-coordinated. A coordinated MAC protocol operates with explicit coordination among the nodes and is generally associated with coordinators, channel access schedules and clusters. A noncoordinated MAC protocol, on the other hand, operates without any explicit coordination among the nodes in the network. For example, IEEE 802.11 is a non-coordinated

Abstract ID: 589. This work was supported in part by the University of Rochester Center for Electronic Imaging Systems and in part by Harris Corporation, RF Communications Division.

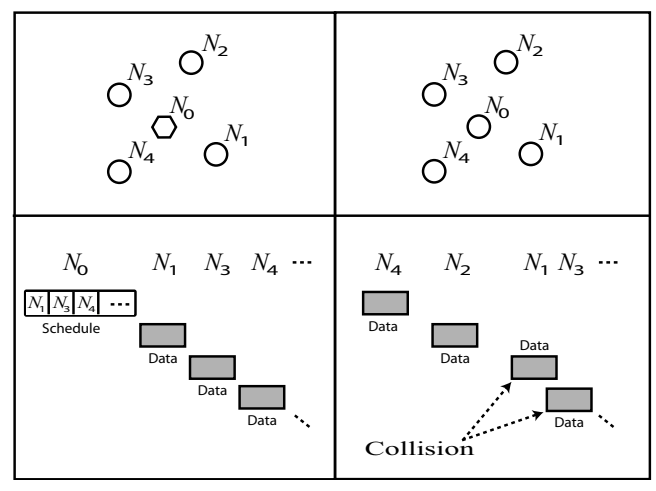

Fig. 1. Illustration of coordinated and non-coordinated MAC protocols. The upper left and right panels show the node distributions for nodes $\mathrm{N}_{0}$ $\mathrm{N}_{4}$. The lower left panel shows the medium access for the coordinated scheme, where node $\mathrm{N}_{0}$ is the coordinator and the channel access is regulated through a schedule transmitted by $\mathrm{N}_{0}$. The lower right panel shows the channel access for the non-coordinated scheme (e.g., CSMA). Overlapping data transmissions of $\mathrm{N}_{1}$ and $\mathrm{N}_{3}$ lead to a collision.

MAC protocol when operating in the broadcast mode (i.e., in broadcasting mode, IEEE 802.11 becomes plain CSMA without any handshaking) [5].

Figure 1 illustrates the channel access mechanism for generic coordinated and non-coordinated MAC protocols. In the coordinated MAC protocol, node $\mathrm{N}_{0}$ is the clusterhead (coordinator) for the portion of the network consisting of five nodes. Channel access is regulated through a schedule that is broadcast by the coordinator. Upon reception of the schedule, nodes transmit their data at their allocated time, and thus collisions among nodes within the same cluster are eliminated. Time is organized into cyclic time frames, and the transmission schedule is dynamically updated at the beginning of each time frame. IEEE 802.15.3 is a recent example of such a coordinated MAC protocol [5]. In the non-coordinated MAC protocol, each node determines its own transmission time based on feedback obtained through carrier sensing on the channel. Thus, conflicts in data transmission attempts (i.e., collisions, capture) are unavoidable in the non-coordinated scheme.

IEEE 802.11 is a well-known example of a noncoordinated MAC protocol when it is used for broadcasting. MH-TRACE (Multi-Hop Time Reservation Using Adaptive 
Control for Energy Efficiency) is a recent example of a coordinated MAC protocol that relies on control packet exchanges for its operation. A comparative evaluation of IEEE 802.11 and MH-TRACE for real-time data broadcasting using a perfect channel showed that the performance of MH-TRACE is better than IEEE 802.11 in terms of throughput and energy efficiency under various network conditions [6]. However, due to the relatively complicated design of MH-TRACE, which relies on robust control packet exchange, the advantages of MH-TRACE over IEEE 802.11 are questionable under a realistic channel error model.

In our previous work we presented a comparative performance evaluation of IEEE 802.11 and MH-TRACE when they are utilized for single hop data broadcasting. The overall performance (e.g., QoS, energy dissipation) of these protocols is directly determined by the performance of the MAC protocol for a relatively low bit error rate (BER) level (i.e., BER $=10^{-4}$ ) through ns-2 simulations [7]. We used a single-hop broadcasting scenario to clearly assess the performance of the MAC layer without being affected by the upper layers. Our analysis revealed that the performance of MH-TRACE is still better than the performance of IEEE 802.11 in terms of throughput and energy efficiency for a channel BER of $10^{-4}$.

In this study we present an analysis of the effects of channel noise for IEEE 802.11 and MH-TRACE for a wide range of BER levels through mathematical models supported by ns-2 simulations.

\section{BACKGROUND}

In this section we present an overview of IEEE 802.11 and MH-TRACE when they are used for single-hop data broadcasting.

\section{IEEE 802.11}

In broadcasting mode, IEEE 802.11 uses $p$-persistent CSMA with a constant defer window length (i.e., the default minimum defer period) [5]. When a node has a packet to broadcast, it picks a random defer time and starts to sense the channel. When the channel is sensed idle, the defer timer counts down from the initially selected defer time at the end of each time slot. When the channel is sensed busy, the defer timer is not decremented. Upon the expiration of the defer timer, the packet is broadcast.

\section{MH-TRACE}

Multi-Hop Time Reservation Using Adaptive Control for Energy Efficiency (MH-TRACE) is a MAC protocol designed for energy-efficient real-time data broadcasting [6].

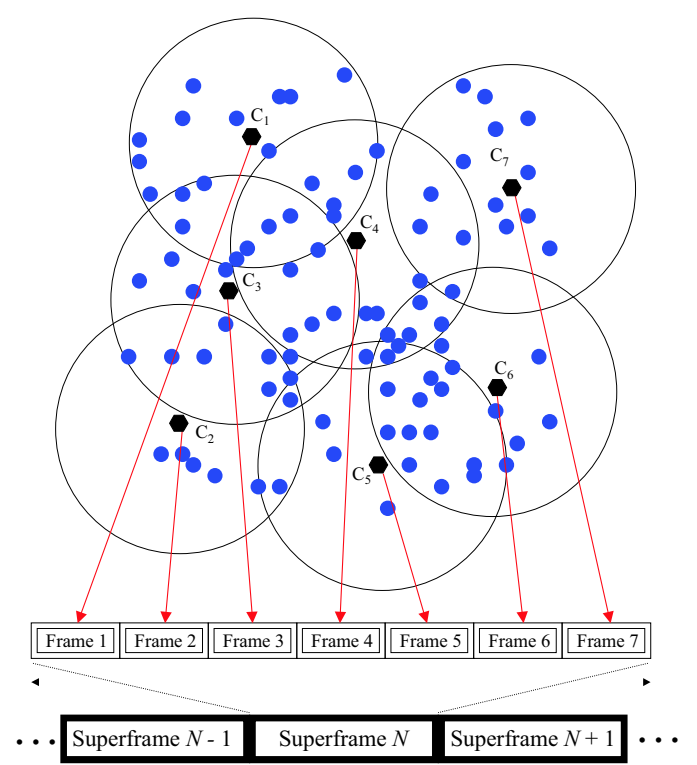

Fig. 2. A snapshot of MH-TRACE clustering and medium access for a portion of an actual distribution of mobile nodes. Nodes $\mathrm{C}_{1}-\mathrm{C}_{7}$ are clusterhead nodes.

Figure 2 shows a snapshot of MH-TRACE clustering and medium access. In MH-TRACE, the network is partitioned into overlapping clusters through a distributed algorithm. Time is organized into cyclic constant duration superframes consisting of several frames. Each clusterhead chooses the least noisy frame to operate within and dynamically changes its frame according to the interference level of the dynamic network. Nodes gain channel access through a dynamically updated and monitored transmission schedule created by the clusterheads.

Each frame consists of a control sub-frame for transmission of control packets and a contention-free data sub-frame for data transmission (see Figure 3). Beacon packets are used for the announcement of the start of a new frame; Clusterhead Announcement (CA) packets are used for reducing co-frame cluster interference; contention slots are used for initial channel access requests; the header packet is used for announcing the data transmission schedule for the current frame; and Information Summarization (IS) packets are used for announcing the upcoming data packets. IS packets are crucial in energy saving. Each scheduled node transmits its data at the reserved data slot.

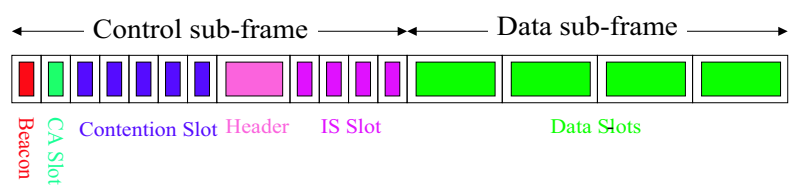

Fig. 3. MH-TRACE frame structure. 
TABLE I

SIMULATION PARAMETERS

\begin{tabular}{|l|l|l|}
\hline Acronym & Description & Value \\
\hline$T_{S F}$ & Superframe duration & $25.172 \mathrm{~ms}$ \\
$N_{F}$ & Number of frames & 7 \\
$N_{D S}$ & Number of data slots per frame & 7 \\
$N_{C}$ & Number of cont. slots per frame & 6 \\
$T_{H}$ & Header slot duration & $92 \mathrm{~s}$ \\
$T_{B, C A, C, I S}$ & Beacon, CA, Cont., IS slot dur. & $32 \mathrm{~s}$ \\
$T_{D}$ & Data slot duration & $432 \mathrm{~s}$ \\
$N / A$ & Data packet size & $104 \mathrm{~B}$ \\
$N / A$ & Header packet size & $4-18 \mathrm{~B}$ \\
$N / A$ & All other control packet size & $4 \mathrm{~B}$ \\
$T_{d r o p}$ & Packet drop threshold & $50 \mathrm{~ms}$ \\
$T_{V F}$ & Voice packet generation period & $25.172 \mathrm{~ms}$ \\
$D_{T r}$ & Transmission range & $250 \mathrm{~m}$ \\
$D_{C S}$ & Carrier Sense range & $507 \mathrm{~m}$ \\
\hline
\end{tabular}

In MH-TRACE, nodes switch to sleep mode whenever they are not involved in data transmission or reception, which saves the energy that would be wasted in idle mode or in carrier sensing. Instead of frequency division or code division, MH-TRACE clusters use the same spreading code or frequency, and inter-cluster interference is avoided by using time division among the clusters to enable each node in the network to receive all the desired data packets in its receive range, not just those from nodes in the same cluster. Thus, MH-TRACE clustering does not create hard clustersthe clusters themselves are only used for assigning time slots for nodes to transmit their data. For a more complete description of MH-TRACE, the reader is referred to [6].

\section{ANALYTICAL MODEL}

In this section we develop an analytical model to estimate the performance of MH-TRACE as a function of BER. In our analysis we do not consider any error correction scheme, thus, if there is at least one bit error within a packet, then that packet is discarded. Random packet errors are independently introduced at the receivers.

\section{BASIC MODEL}

To minimize the number of parameters in the model, first we consider a fully-connected network with a small number of static nodes. The number of data slots in one superframe is high enough to support all of the nodes in the network (see Table I). When there are no channel errors, all nodes should be able to transmit and receive without any packet drops or collisions. There will be only one clusterhead in the network due to the fact that there cannot be two clusterheads that can hear each other directly.

The number of data packets generated per node per second, $\left(D P_{\text {node }}\right)$, is equal to the packet rate $\left(R_{\text {packet }}\right)$ of

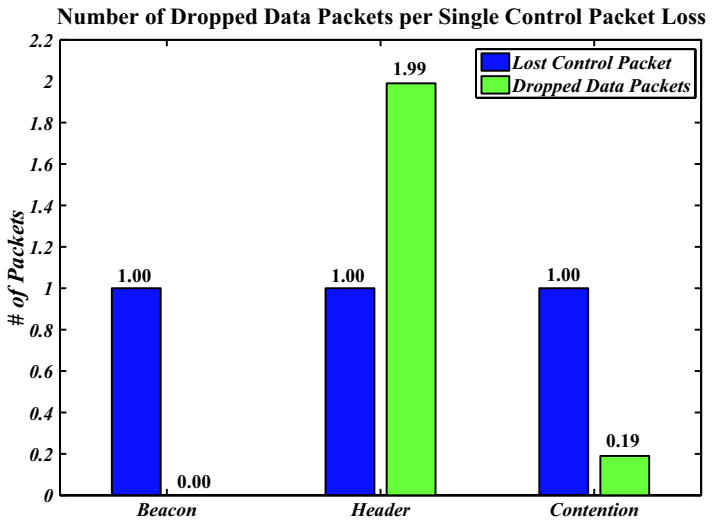

Fig. 4. MH-TRACE performance degradation in terms of dropped data packets for beacon, header, and contention packet losses.

MH-TRACE (i.e., one packet per superframe time $\left.\left(1 / T_{s f}\right)\right)$.

$$
D P_{\text {node }}=R_{\text {packet }}=\frac{1}{T_{s f}}
$$

$D P_{\text {node }}$ represents the number of data packets generated by a single node in the network and can be regarded as the maximum number of packets a node can transmit given that it has full access to a perfect channel whenever it needs. However a lossy channel will cause packet drops and therefore the throughput of the network will drop accordingly.

In Figure 4, the corresponding throughput losses due to corrupted beacon, header and contention packets are given to illustrate the impact of the particular control packet on overall protocol performance. In these results only the specified control packets are lost due to channel errors and all the other packets are not affected [7].

As can be seen from the figure, header packets are vital to MH-TRACE and are the packets whose loss has the most impact on the performance of MH-TRACE. Loss of contention packets cause 10 times less loss in data packets than loss of header packets (0.19). Finally, for each beacon packet dropped, only 0.0015 data packets are dropped. Like beacon packet losses, losses of other control packets (e.g., IS, CA) do not significantly affect the throughput of the network. Thus, the header and contention packets are the only control packets whose loss due to channel noise significantly affect the network performance.

Therefore, we can write the equation for the transmit throughput of a single node (i.e., transmit throughput per node per second $\left.T_{\text {node }}\right)$ in terms of the data packets dropped before transmission due to lost header packets $\left(D P L_{H}\right)$ and contention $\left(D P L_{C}\right)$ packets:

$$
T_{\text {node }}=D P_{\text {node }}-D P L_{H}-D P L_{C}
$$


Both $\left(D P L_{H}\right)$ and $\left(D P L_{C}\right)$ can be expanded as the product of three parts.

- Number of data packets dropped per header/contention packet loss $\left(D P L_{p e r H} / D P L_{p e r C}\right)$.

- Number of header/contention packets sent to a node/clusterhead per second ( $\left.H P_{\text {node }} / C P_{\text {node }}\right)$.

- Probability of dropping a header/contention packet $\left(P_{H} / P_{C}\right)$.

As contention packets are relatively short (4 bytes), they are less likely to be dropped than header packets (16 bytes for 6 broadcasting nodes). Furthermore, since the sources are continuous bit rate and MH-TRACE utilizes automatic channel access renewal, once a node gets channel access, it will not loose it and thus will not need to transmit contention packets for the rest of the simulation time. Moreover, the number of dropped data packets per lost header packet is 10 times larger than the number of dropped data packets per lost contention packet, as shown in Figure 4. Therefore it is reasonable to assume that the effect of losing contention packets can be neglected. Based on this assumption, the transmit throughput per node per second becomes:

$$
\begin{gathered}
T_{\text {node }}=D P_{\text {node }}-D P L_{H} \\
T_{\text {node }}=\frac{1}{T_{s f}}-D P L_{\text {per } H} \times H P_{\text {node }} \times P_{H} .
\end{gathered}
$$

In Equation (4), $D P L_{\text {per } H}$ is a constant (1.99) and $H P_{\text {node }}$ is equal to $D P_{\text {node }}$ since each node receives one header per super frame from its clusterhead. Finally $P_{H}$ depends on the length of the header packet $L_{H}$ and is calculated from the Bit Error Rate (BER) of the channel.

$$
P_{H}=\left\{1-(1-B E R)^{L_{H}}\right\} .
$$

Therefore,

$$
\begin{aligned}
& T_{\text {node }}=\frac{1}{T_{s f}}-1.99 \times \frac{1}{T_{s f}} \times\left\{1-(1-B E R)^{L_{H}}\right\} \\
& T_{\text {node }}=\frac{1}{T_{s f}} \times\left[1-1.99 \times\left\{1-(1-B E R)^{L_{H}}\right\}\right] .
\end{aligned}
$$

In order to get the number of received packets per second in the network we need to multiply the transmit throughput per node per second with the number of neighboring nodes $N-1$ (note that all the nodes can hear each other in this network). Moreover, each data packet is received with a probability $P_{D}$, which is the probability that a data packet (with length $L_{D}=104$ bytes) goes through the channel with no error at a given BER. Accordingly, the receive throughput per node per second $(T)$ becomes:

$$
T=(N-1) \times T_{\text {node }} \times(1-B E R)^{L_{D}} .
$$

Note that the receive throughput per node per second of IEEE 802.11 is simply equal to $\frac{N-1}{T_{s f}} \times(1-B E R)^{L_{D}}$ since in CSMA-type protocols such IEEE 802.11 in broadcasting

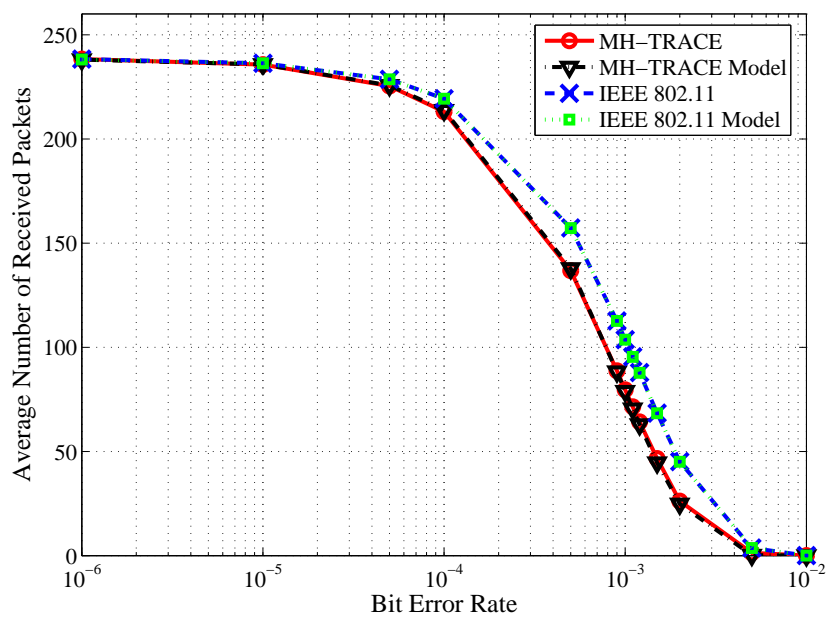

Fig. 5. Average number of received packets per node per second versus bit error rate (BER).

mode, only data packets are sent through the lossy channel and the throughput is determined by the BER of the channel and length of a data packet.

We used the ns-2 simulator to validate the analytical model. The channel rate is set to $2 \mathrm{Mbps}$, and all nodes have a CBR (Constant Bit Rate) data source with $32 \mathrm{Kbps}$ data rate, which corresponds to one voice packet per superframe. The simulations are run for $1000 \mathrm{~s}$ and repeated with the same parameters five times.

In Figure 5 the analytical model for MH-TRACE and IEEE 802.11 are plotted against increasing BER. Also the simulation results are included for both protocols to demonstrate the accuracy of the models. The throughput of MH-TRACE drops by almost $50 \%$ at a BER around $7 \times 10^{-4}$. On the other hand, IEEE 802.11 retains almost $55 \%$ of its initial throughput at the same BER (note that the initial throughputs of both protocols are the same). This difference can be translated into the fact that IEEE 802.11 performs $10 \%$ better than MH-TRACE, which experiences a worse performance degradation due to lost coordination packets [7].

These results show that the analytical model proposed to estimate the throughput of MH-TRACE is quite accurate. The model captures the fact that coordinated MAC protocols are more vulnerable than non-coordinated MAC protocols to channel noise due to their dependence on the robustness of the control traffic. However, in our model, we treated the clusterhead as a regular node inside the network, but in reality, a clusterhead would not drop any data packets due to lost header packets since the clusterhead is the one generating the header packets. Therefore, our model slightly underestimates the throughput of MH-TRACE by treating the clusterhead as an ordinary node. 


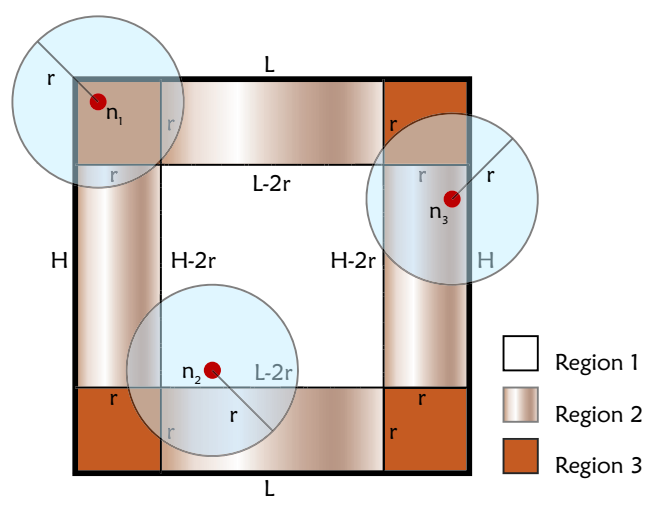

Fig. 6. Rectangular field partitioned into three different regions.

\section{GENERAL MODEL}

In this section, we consider a rectangular field $(L \times H)$ in which a certain number of nodes $(N)$, which have a communication radius $(r)$, are randomly deployed. We use a statistical voice source model that classifies speech into spurts and gaps (i.e., gaps are the silent moments during a conversation). During gaps, no data packets are generated, and during spurts, data packets are generated at $32 \mathrm{Kbps}$ data rate. Both spurts and gaps are exponentially distributed statistically independent random variables, with means $\eta_{s}=$ $1.0 s$ and $\eta_{g}=1.35 s$, respectively [8].

Our approach to this more complex model will be basically the same as before. We begin by calculating the transmit throughput per node per second $\left(T_{\text {node }}\right)$ when the channel is perfect. In addition to Equation (7) we need a term that captures the effect of the voice source model. This term can easily be represented with the ratio of spurts to the whole conversation $(\eta)$. Therefore, we can write $T_{\text {node }}$ as in Equation (9).

$$
\begin{aligned}
T_{\text {node }} & =\frac{1}{T_{s f}}\left[1-1.99\left\{1-(1-B E R)^{L_{H}}\right\}\right][\eta] \\
& =\frac{1}{T_{s f}}\left[1-1.99\left\{1-(1-B E R)^{L_{H}}\right\}\right]\left[\frac{\eta_{s}}{\eta_{s}+\eta_{g}}\right]
\end{aligned}
$$

After obtaining the expression for the transmit throughput per node per second, we have to find an expression for the average number of nodes within the communication rage of a given node (i.e., the average number of neighbors for a given node). In Figure 6, the rectangular field is partitioned into three different regions according to the coverage characteristic of a node in a particular region. For example, a node inside region 1 (e.g., $\left.n_{2}\right)$ has its full coverage within the boundaries of the field. Therefore, any node inside region 1 utilizes $100 \%$ of its total coverage. Whereas nodes inside regions 2 and 3 (e.g., $n_{1}$ and $n_{3}$ )
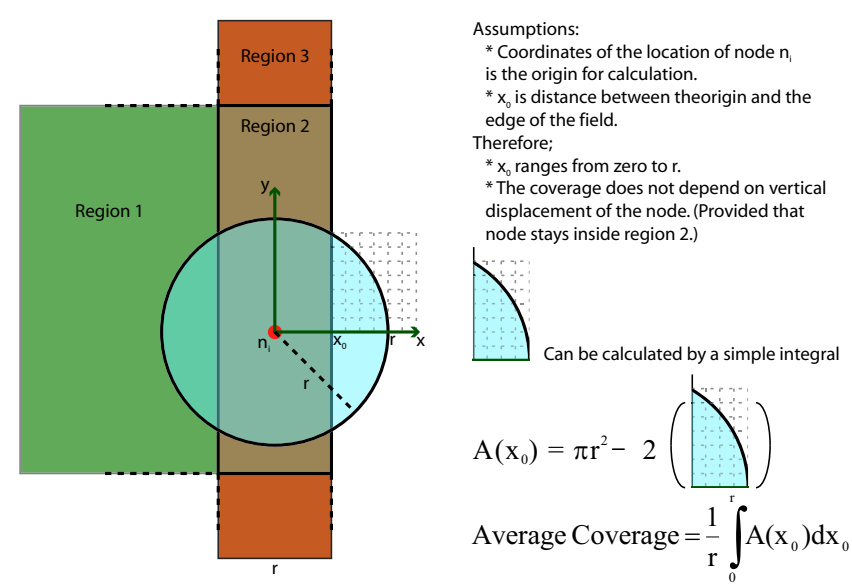

Fig. 7. Calculation of the percentage coverage of a node inside region 2.

have a part of their coverage outside the field of interest and consequently the average percentage coverage for these nodes is less than $100 \%$. Finding the percentage coverage for each region will lead us to the average number of neighbors.

We start the derivation of the percentage with region 2 . In Figure 7 the approach we used for obtaining the percentage is given. The area of the piece of circle shaded in Figure 7 can be expressed as follows:

$$
\begin{aligned}
I & =\int_{x_{0}}^{r} \sqrt{r^{2}-x^{2}} d x \\
& =\frac{\pi}{4} r^{2}-\frac{x_{0}}{2} \sqrt{r^{2}-x_{0}^{2}}-\frac{r^{2}}{2} \arcsin \left(\frac{x_{0}}{r}\right) .
\end{aligned}
$$

Thus, the average coverage for region $2\left(\alpha_{2}\right)$ becomes,

$$
\begin{aligned}
\alpha_{2} & =\frac{1}{r} \int_{0}^{r} A\left(x_{0}\right) d x_{0} \\
& =\frac{1}{r} \int_{0}^{r}\left(\pi r^{2}-2 I\left(x_{0}\right)\right) d x_{0} \\
& =\pi r^{2}-\frac{2}{3} r^{2} .
\end{aligned}
$$

After obtaining the average coverage as in Equation (11), we can easily calculate the percentage coverage of region 2 $\left(\sigma_{2}\right)$.

$$
\sigma_{2}=\frac{\alpha_{2}}{\pi r^{2}}=1-\frac{2}{3 \pi}
$$

Next we derive the average coverage for region $3\left(\sigma_{3}\right)$. The area in question is divided into three parts (see Figure 8). According to this partitioning we have $A=\pi r^{2}-$ $\left(A_{1}+A_{2}-A_{3}\right)$, which is the coverage for a node inside region 3. The integrals for $A_{1}$ and $A_{2}$ are the same as $I$ given in Equation (10) and can be expressed as $2 I\left(x_{0}\right)$ and $2 I\left(y_{0}\right)$, respectively. 


$$
\begin{aligned}
A_{3}= & \int_{x_{0}}^{\sqrt{r^{2}-y_{0}^{2}}}\left(\sqrt{r^{2}-x^{2}}-y_{0}\right) d x \\
= & -\frac{y_{0} \sqrt{r^{2}-y_{0}^{2}}}{2}+\frac{r^{2} \arcsin \left(\frac{\sqrt{r^{2}-y_{0}^{2}}}{r}\right)}{2} \\
& -\frac{x_{0} \sqrt{r^{2}-x_{0}^{2}}}{2}-\frac{r^{2} \arcsin \left(\frac{x_{0}}{r}\right)}{2}+y_{0} x_{0} .
\end{aligned}
$$

After obtaining $A_{3}$, we can calculate the average coverage $\alpha_{3}$ by taking the average of $A$.

$$
\begin{aligned}
\alpha_{3} & =\frac{1}{r^{2}} \int_{0}^{r} \int_{0}^{r} A\left(x_{0}, y_{0}\right) d x_{0} d y_{0} \\
& =\pi r^{2}-\frac{29}{24} r^{2} .
\end{aligned}
$$

Thus, $\sigma_{3}$ becomes:

$$
\sigma_{3}=\frac{\alpha_{3}}{\pi r^{2}}=1-\frac{29}{24 \pi}
$$

This is the last percentage coverage we needed to calculate the overall percentage coverage $(\sigma)$, or the average number of nodes within the range of a given node inside the rectangular field. Below we give the resulting $\sigma$ in terms of the communication radius $r$, the length of the field $L$ and the height of the field $H$.

$$
\sigma=\frac{\sigma_{1}(L-2 r)(H-2 r)+2 \sigma_{2}(H+L-4 r) r+4 \sigma_{3} r^{2}}{L H}
$$

This expression can be used to calculate the average number of neighboring nodes $\left(N_{N}\right)$ for a node inside of a rectangular field by multiplying $\sigma$ with $\pi r^{2}$ (i.e., the coverage of a node with communication radius $r$ ) and the node density $\left(\frac{(N-1)}{L H}\right)$. Note that there are $N-1$ nodes remaining that can be neighbors.

$$
N_{N}=\frac{(N-1) \sigma \pi r^{2}}{L H}
$$

Now, we can combine Equation (17) with Equation (9) to get the receive throughput per node per second $T$.

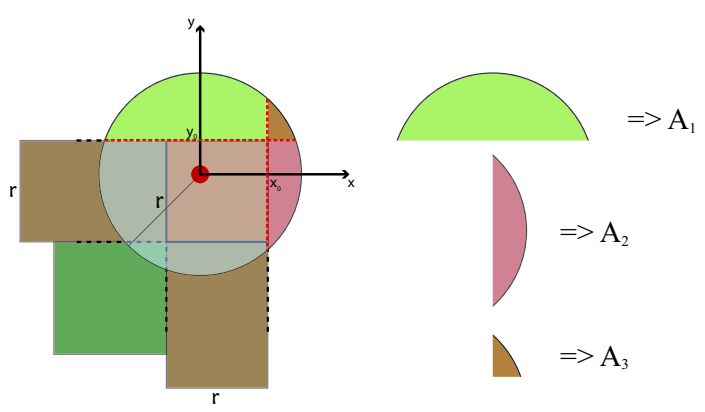

Fig. 8. Calculation of the percentage coverage of a node inside region 3.

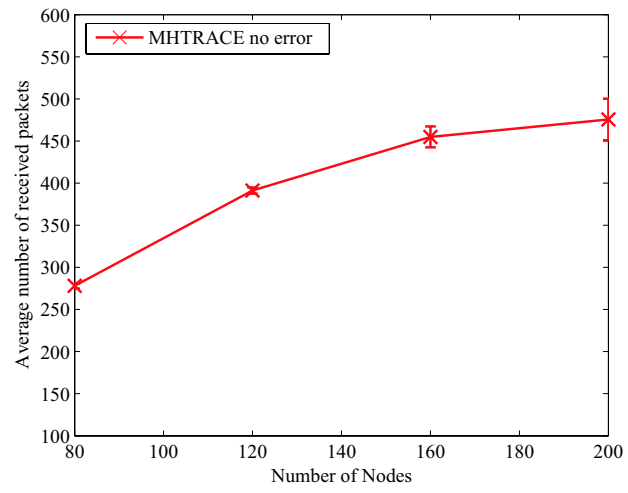

Fig. 9. Average number of received packets per node per second versus number of nodes.

$$
T=N_{N} \times T_{\text {node }} \times(1-B E R)^{L_{D}}
$$

According to our model, given that we have a constant simulation area and the same traffic model, throughput increases as the number of nodes in the network increases. In other words, the model suggests that throughput increases linearly with increasing node density. However, our previous work showed that throughput per node per second goes into saturation as the number of nodes in the network increases (see Figure 9). This trend is a result of packet collisions and drops emerging from mobility and increased contention for channel access [7]. According to this fact, we have to modify our initial throughput value (throughput when there is a perfect channel) in order to get a more accurate model for throughput. Since it is extremely challenging to model the dynamical behavior in Figure 9 analytically, the initial throughput values are calibrated according to feedback from simulation results.

\section{SIMULATIONS}

In this section we present simulations to demonstrate the validity of the analytical results. The simulation parameters are given in Table II.

TABLE II

Simulation Setup

\begin{tabular}{ccc}
\hline PARAMETER & SET 1 & SET 2 \\
\hline Number of Nodes & 100 & 100 \\
Simulation Area & $1000 \mathrm{~m} \times 1000 \mathrm{~m}$ & $1000 \mathrm{~m} \times 1000 \mathrm{~m}$ \\
Simulation Time & $200 \mathrm{~s}$ & $200 \mathrm{~s}$ \\
Protocol & MH-TRACE/ & MH-TRACE/ \\
& IEEE 802.11 & IEEE 802.11 \\
Number of Repetition & 10 & 10 \\
Node Mobility & Stationary & Mobile \\
\hline
\end{tabular}




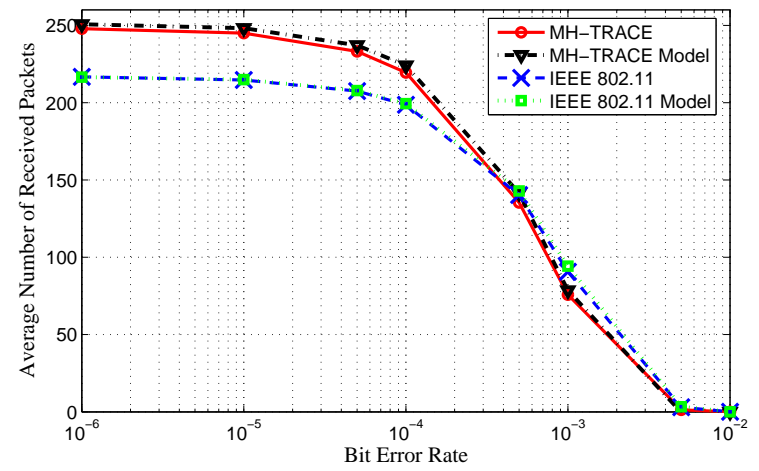

Fig. 10. SET 1 (Stationary nodes): Average number of received packets per node per second versus bit error rate (BER).

The results of SET 1 simulations are given in Figure 10, which presents the receive throughput per node per second for MH-TRACE and IEEE 802.11 along with the analytical resluts as a function of the BER. MH-TRACE throughput is obtained from the analytical model in Equation (18). The guideline for IEEE 802.11 is obtained by using the probability of successful data packet transmission ((1 $B E R)^{L_{D}}$ ) and the initial throughput value.

When we look at Figure 10, we see that the throughput of MH-TRACE is higher than IEEE 802.11 for low BER (i.e., BER $<10^{-4}$ ). However, as BER increases, MH-TRACE throughput decreases below the throughput of IEEE 802.11. Although this performance loss seems to be a drawback for any coordinated protocol, we have to keep in mind that the BER value at which the performance of MH-TRACE becomes worse than IEEE 802.11 causes almost $30 \%$ of the data packets to be dropped.

For SET 2 simulations, nodes are mobile and we use a Random Way-Point mobility model with node speeds chosen from a uniform distribution between $0.0 \mathrm{~m} / \mathrm{s}$ and 5.0 $\mathrm{m} / \mathrm{s}$ (the average pace of a marathon runner) with zero pause time. As shown in Figure 11, the average throughputs for MH-TRACE and IEEE 802.11 are almost $40 \%$ more than

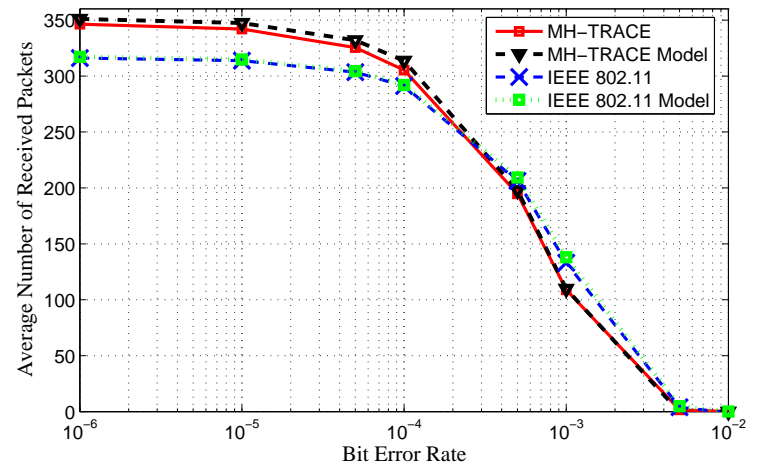

Fig. 11. SET 2 (Mobile nodes): Average number of received packets per node per second versus bit error rate (BER). the throughputs with stationary nodes. This is a result of the mobility model which makes the nodes accumulate in the middle of the field instead of distributing them uniformly [9], providing a larger average number of neighbors $\left(N_{N}\right)$ than in the stationary case. Therefore the initial throughput value is calibrated. Note that after adjusting the initial value the form of the curve tracks the simulation results closely.

\section{CONCLUSIONS}

In this paper we proposed an analytical model for the throughput of MH-TRACE. Parameters such as network area, number of nodes and BER of the channel are included in the model. Moreover, we derived an expression to determine the average number of single-hop neighbors. The impact of channel errors on the performance of MH-TRACE and IEEE 802.11, which are examples of coordinated and non-coordinated MAC protocols, respectively, are estimated by using this model. We also presented ns- 2 simulations to demonstrate the validity of the model. As expected, the performance of MH-TRACE is better than IEEE 802.11 for low-mid BER levels. However, for extremely high BER rates IEEE 802.11 performance is better than MH-TRACE due to the dependence of MH-TRACE on the robustness of the control packet traffic. However, for higher data rates or node densities, we expect MH-TRACE to perform better than IEEE 802.11 even under very high BER levels due to its coordinated channel access mechanism.

\section{REFERENCES}

[1] W. Stallings. Wireless Communications and Networks. Prentice-Hall, 2002.

[2] A. Chandra, V. Gummalla, and J. O. Limb. Wireless medium access control protocols. IEEE Communications Surveys and Tutorials, 3:215,2000 .

[3] P. Mohapatra, J. Li, and C. Gui. QoS in mobile ad hoc networks. IEEE Wireless Communications, 10(3):44-52, June 2003.

[4] D. A. Maltz, J. Broch, and D. B. Johnson. Lessons from a full-scale multihop wireless ad hoc network testbed. IEEE Personal Commun. Mag., 8(1):8-15, Feb 2001.

[5] T. Cooklev. Wireless Communication Standarts. IEEE Press, 2004.

[6] B. Tavli and W. B. Heinzelman. MH-TRACE: Multi hop time reservation using adaptive control for energy efficiency. IEEE Journal on Selected Areas of Communications, 22(5):942-953, June 2004.

[7] T. Numanoglu, B. Tavli, and W. B. Heinzelman. The effects of channel errors on coordinated and non-coordinated medium access control protocols. IEEE International Conference on Wireless and Mobile Computing, 2005 (Available at http://www.ece.rochester.edu/ numanogl/wimob_05.pdf).

[8] D. J. Goodman and S. X. Wei. Efficiency of packet reservation multiple access. IEEE Trans. Vehic. Tech., 40(1):170-176, Feb 1991.

[9] W. Navidi and T. Camp. Stationary distributions for the random waypoint mobility model. Technical Report MCS-03-04, The Colorado School of Mines, April 2003. 